\title{
Helicobacter pylori cytotoxin-associated gene A impairs the filtration barrier function of podocytes via p38 MAPK signaling pathway
}

\author{
Man Yang, Ling Wang, Li-jie Gu and Wei-jie Yuan ${ }^{凶}$ \\ Department of Nephrology, Shanghai General Hospital, Shanghai Jiao Tong University, Shanghai 200080, China
}

\begin{abstract}
Helicobacter pylori ( $\mathrm{Hp})$ specific antigens were found deposited in the glomeruli in some kidney diseases. However, the underlying molecular mechanisms remain to be elucidated. The aim of this study was to investigate the effect of cytotoxin associated gene A protein (CagA), a key virulence factor of $\mathrm{Hp}$, on mouse podocytes. Cells were cultured and treated with recombinant CagA protein. The expression of the tight junction protein ZO-1 and p38 MAPK signaling pathway activation were measured with real-time RT-PCR and western blotting. The filtration barrier function of podocytes was evaluated with albumin influx assay. CagA decreased the expression and membrane distribution of ZO-1, impaired the filtration barrier function of podocytes, while activating p38 MAPK signaling pathway in these cells. Selective p38 MAPK inhibition partly prevented CagA-induced filtration barrier dysfunction of podocytes through ameliorating ZO-1 downregulation. Taken together, the results suggested that CagA, at least via p38 MAPK signaling pathway, may induce podocyte injury. Anti-Hp therapy may be beneficial for the treatment of kidney diseases related to $\mathrm{Hp}$ antigen deposition.
\end{abstract}

Key words: CagA, ZO-1, p38 MAPK, podocyte, proteinuria

Received: 22 May, 2016; revised: 15 August, 2016; accepted: 17 August, 2016; available on-line: 12 August, 2017

e-mail: ywj4169@163.com

Abbreviations: Hp, Helicobacter pylori; CagA, cytotoxin associated gene $A$ protein; VacA, vacuolating toxin $A$; $M N$, membranous nephropathy; HSPN, Henoch Schonlein Purpura nephritis; LN, lupus nephritis; ECL, enhanced chemiluminescence; MALT, mucosa-associated lymphoid tissue

\section{INTRODUCTION}

Helicobacter pylori (Hp), a gram-negative bacterium, was reported to infect more than $50 \%$ of the population in Asia (Eusebi et al., 2014). A number of virulence factors of $\mathrm{Hp}$ were identified such as cytotoxin-associated gene A ( CagA), vacuolating toxin A ( VacA) (Testerman \& Morris, 2014). Among them, CagA is the most important and best-studied virulence factor, responsible for alterations in multiple intracellular signaling pathways and consequently exerting a great influence on host cell function (Censini et al, 1996; Covacci et al, 1993; Testerman \& Morris, 2014).

Hp infection is not only associated with some gastrointestinal diseases such as peptic ulcer disease, gastric cancer, but also plays a role in the pathogenesis of several extragastric diseases such as cardiovascular diseases, diabetes mellitus and autoimmune diseases (Franceschi et al., 2014). Recently, some evidence suggested a poten- tial connection between Hp infection and particular kidney diseases. In patients with membranous nephropathy (MN), Henoch Schonlein Purpura nephritis (HSPN) or lupus nephritis ( $\mathrm{LN})$, Hp-specific antigens were found deposited along the glomerular capillary walls (Nagashima et al., 1997; Yang et al., 2009; Li et al., 2013). While $\mathrm{Hp}$ eradication treatment induced significant remission of proteinuria in patients with primary glomerulonephritis including $\mathrm{MN}$, the underlying mechanisms of the $\mathrm{Hp}$ specific antigens deposition remain unknown (Dede et al., 2015).

Podocyte injury plays a crucial role in the development of proteinuria (Brinkkoetter et al., 2013). Tight junction protein ZO-1, an important component of podocyte slit diaphragm, is indispensable for maintaining the integrity of glomerular filtration barrier (Itoh et al., 2014). The suppression of ZO-1 impairs the formation of podocyte slit diaphragm and consequently triggers the onset of proteinuria (Itoh et al., 2014). Whether the Hp-specific antigens deposited in glomeruli may induce abnormal expression and/or distribution of ZO-1 and subsequent podocyte injury remains to be elucidated. Therefore, the present study was designed to investigate the effect of $\mathrm{CagA}$, the key virulence factor of $\mathrm{Hp}$, on podocyte injury and proteinuria and its possible mechanisms.

\section{MATERIALS AND METHODS}

Cell culture and experimental protocol. The conditionally immortalized mouse podocyte cell line was kindly provided by Dr Peter Mundel (Mount Sinai School of Medicine, New York, NY, USA) and cultured as described previously (Jing et al., 2015). Recombinant CagA protein which originates from $\mathrm{Hp}$ strain ATCC 43504 and contains three EPIYA motifs, was purchased from Shanghai Linc- Bio Science Co. Ltd (Shanghai, China). For dose-dependent experiments, differentiated podocytes were incubated with different concentration $(0$, 5,10 and $20 \mu \mathrm{g} / \mathrm{ml}$ ) of recombinant CagA protein for $72 \mathrm{~h}$; for time-dependent experiments, cells were incubated with the optimal concentration of recombinant CagA protein for 12, 24, 48 and $72 \mathrm{~h}$. Then the optimal concentration and incubation time were used in the subsequent experiments. To investigate the role of p38 MAPK signaling pathway in podocyte injury, differentiated podocytes were pretreated with $5 \mu \mathrm{M}$ SB203580, a p38 inhibitor (ApexBio, TX, USA) for $1 \mathrm{~h}$ prior to being incubated with recombinant $\mathrm{CagA}$ protein.

CCK-8 assay. Cell viability was evaluated with CCK8 assay (Signalway Antibody, MA, USA). 3000 cells per 
well were plated in 96-well plate, cultured until attachment, then treated with different concentration $(0,5$, 10 and $20 \mu \mathrm{g} / \mathrm{ml}$ ) of recombinant CagA protein. At 0 $\mathrm{h}$ or $72 \mathrm{~h}$ of the treatment, $10 \mu \mathrm{l}$ of CCK- 8 solution was added to each well of the 96-well plate and the plate was incubated for $1 \mathrm{~h}$ at $37^{\circ} \mathrm{C}$. Then the absorbance was measured at $450 \mathrm{~nm}$ using a spectrophotometer.

Real time RT-PCR. Total RNA was extracted from cells using Trizol reagent (Invitrogen, CA, USA). cDNA synthesis from total RNA was performed using the RevertAid First Strand cDNA Synthesis Kit (Thermo Scientific Fermentas, MA, USA). Resulting cDNA (1 $\mu \mathrm{g})$ was amplified in real time, in $25 \mu \mathrm{l}$ reaction mixture containing SYBR Green PCR Master Mix (Applied Biosystems, CA, USA), appropriate primer pairs and water using ABI 7300 thermocycler (Applied Biosystems, CA, USA). The reaction mixtures were incubated at $95^{\circ} \mathrm{C}$ for $10 \mathrm{~min}$, followed by 40 cycles of $95^{\circ} \mathrm{C}$ for $15 \mathrm{~s}$ and $60^{\circ} \mathrm{C}$ for $45 \mathrm{~s}$. The expression of target gene was normalized to the reference gene GAPDH in the same cDNA sample. The following primers were used: ZO-1: forward, 5'-AGGCTACCT'T'TGAT'TCTC-3', reverse, 5'-TAGGGCACAGTATTGTATC-3'; p38 MAPK: forward, 5'-GTGTTCACACCCGCAAGGTC-3', reverse, 5'-CGGTCAGCTTCTGGCACTTC-3'; GAPDH: forward, 5'-ATCACTGCCACCCAGAAG-3', reverse, 5'-TCCACGACGGACACATTG-3'. The results were analyzed using $2^{-\triangle \Delta C T}$ method.

Western blotting. Total and membrane proteins were extracted using RIPA buffer (Solarbio, Beijing, China) and the membrane protein extraction kit (Beyotime, Shanghai, China), according to the manufacturer's instructions, respectively. Then the extracted proteins were resolved using SDS-PAGE and transferred to nitrocellulose (NC) membranes (Millipore, MA, USA). The NC membranes were blocked with $5 \%$ non-fat milk and incubated with primary antibodies overnight, followed by incubation with appropriate secondary antibodies for 1 hour at room temperature. The images were visualized using an enhanced chemiluminescence (ECL) detection system (Millipore, MA, USA). The primary antibodies used were as follow: anti-ZO-1 (1:50; Abcam, MA, USA), anti-p38 (1:1000; Cell Signaling Technology, USA), anti-p-p38 (1:1000; Cell Signaling Technology, MA, USA), anti- $\beta$-actin (1:1500; Cell Signaling Technology, MA, USA), and anti-GAPDH (1:1500; Cell Signaling Technology, MA, USA).
Albumin influx assay. To evaluate the filtration barrier function of podocytes, albumin influx assay was performed as described previously (Kumar et al., 2010). Briefly, differentiated podocytes $\left(5 \times 10^{4}\right)$ were seeded onto the collagen-coated transwell filters (0.4- $\mu \mathrm{m}$ pore; Corning, NY, USA) at the top chamber. Then podocytes were serum-starved overnight (for the cell confluency level before CagA treatment see Fig. 1A) and treated with $20 \mu \mathrm{g} / \mathrm{ml}$ of recombinant CagA protein for $72 \mathrm{~h}$. To determine the role of p38 MAPK signaling pathway in the filtration barrier function of podocytes, differentiated podocytes were pretreated with $5 \mu \mathrm{M}$ SB203580 for $1 \mathrm{~h}$ prior to being treated with recombinant CagA protein. After treatment with recombinant CagA protein, podocytes were washed twice with PBS supplemented with $1 \mathrm{mM} \mathrm{MgCl}_{2}$ and $1 \mathrm{mM} \mathrm{CaCl}$ to preserve the cadherin-based junctions. Then the top chamber was refilled with $0.15 \mathrm{ml}$ of RPMI 1640 and the bottom chamber with $1 \mathrm{ml}$ of RPMI 1640 supplemented with $40 \mathrm{mg} / \mathrm{ml}$ of bovine serum albumin. The podocytes were incubated at $37^{\circ} \mathrm{C}$ for $1 \mathrm{~h}$. Then a small aliquot of medium from top chamber was collected and the albumin concentration was mesured using the bicinchoninic acid protein assay kit (Nanjing Jiancheng Bioengineering Institute, Nanjing, China).

Statistical analysis. All the experiments were repeated at least three times. All data were presented as mean \pm S.D. Differences between groups were evaluated by independent Student's $t$-test using SPSS version 16.0 (SPSS Inc., USA). $P<0.05$ was considered significant.

\section{RESULTS}

\section{Dose- and time-dependent effect of CagA on the protein expression of ZO-1 in mouse podocytes}

Within the range of $5-20 \mu \mathrm{g} / \mathrm{ml}, \mathrm{CCK}-8$ assay demonstrated that CagA had no significant influence on cell viability in mouse podocytes (Fig. 1B). However, CagA decreased the protein expression of ZO-1 in mouse podocytes in a dose-dependent manner (Fig. 2A and B). Accordingly, $20 \mu \mathrm{g} / \mathrm{ml}$ was chosen as the optimal concentration of CagA for subsequent studies. When compared to the control group, CagA treatment decreased the protein expression of $\mathrm{ZO}-1$ in a time-dependent

\section{CCK-8 assay}

B
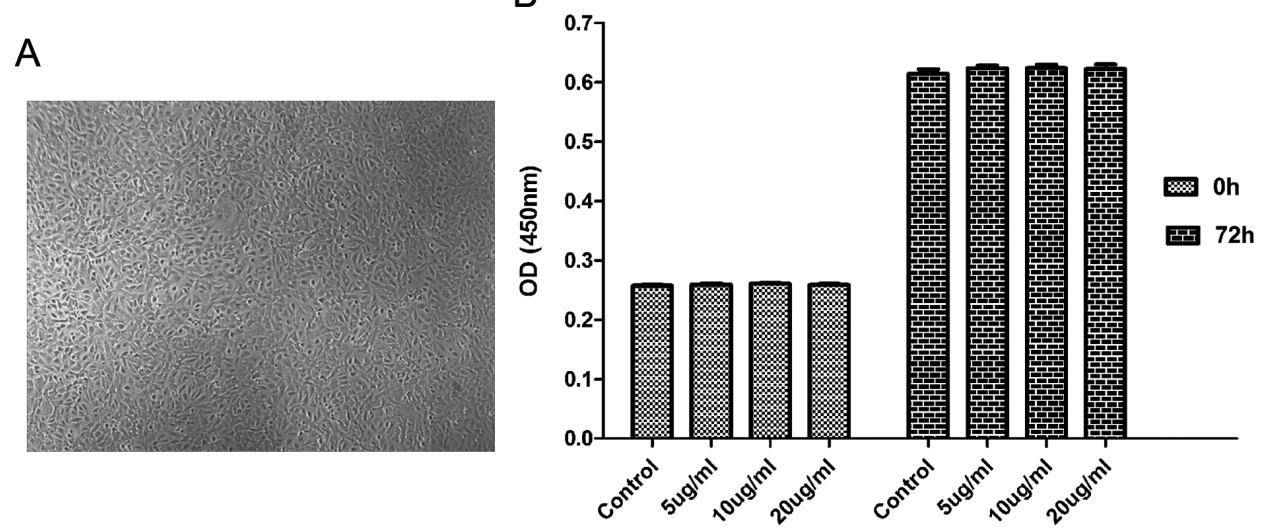

Figure 1. Cell confluency level and CCK-8 assay results.

(A) Podocytes at $100 \%$ confluence before CagA treatment photographed under an inverted microscope. Original magnification, $\times 100$. (B) At $0 \mathrm{~h}$ or $72 \mathrm{~h}$, CCK-8 assay showed that there was no significant difference in cell viability between podocytes treated with different concentration $(0,5,10$ and $20 \mu \mathrm{g} / \mathrm{ml})$ of recombinant CagA protein. 
A
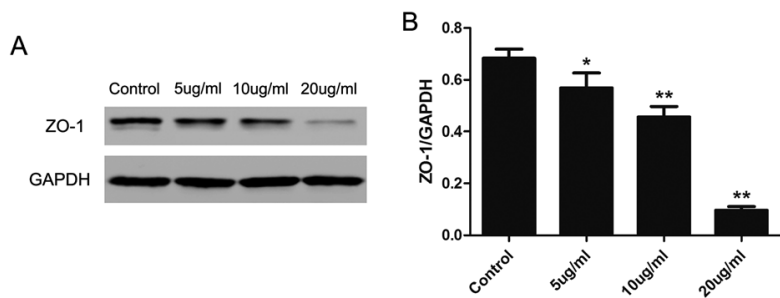

C

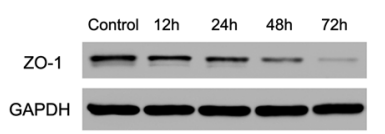

D

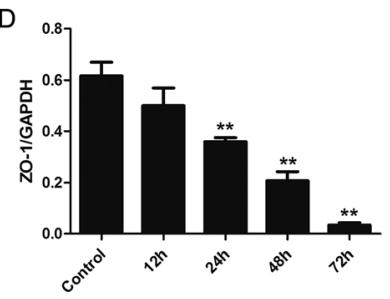

Figure 2. Dose- and time-dependent decrease of ZO-1 protein expression in CagA-treated mouse podocytes.

(A and B) Western blotting (A) and quantitative analysis (B) showed that when compared to control, CagA significantly decreased ZO-1 protein expression in a dose-dependent manner. (C and D) Meanwhile, ZO-1 protein expression was also markedly reduced by CagA treatment in a time-dependent manner. ${ }^{*} p<0.05$, compared to control; ${ }^{* *} p<0.01$, compared to control.

manner from 24 to $72 \mathrm{~h}$, while there was no statistical difference at $12 \mathrm{~h}$ (Fig. 2C and D).

\section{Influence of CagA on the expression of total and membrane ZO-1 as well as on the filtration barrier function of mouse podocytes}

In comparison to the control group, the mRNA expression of ZO-1 was significantly decreased after CagA treatment for $72 \mathrm{~h}$ (Fig. 3A). Consistently, with the mRNA change the protein levels of total and membrane ZO-1 were also downregulated (Fig. $3 \mathrm{~B}$ and C). Moreover, albumin influx assay was used to evaluate the filtration barrier function of mouse podocytes. CagA treatment resulted in an increased albumin influx across the podocyte monolayer at $72 \mathrm{~h}$, which suggested an impaired filtration barrier function of podocytes (Fig. 3D).

\section{Effect of CagA on p38 MAPK signaling pathway in mouse podocytes}

When compared to the control group, the level of p38 MAPK mRNA was significantly increased after CagA treatment for $72 \mathrm{~h}$, while SB203580 pretreatment partly diminished this effect (Fig. 4A). Consistently with the mRNA level change, the protein levels of total and phosphorylated (activated) p38 MAPK were also increased after CagA treatment for 72 h, while SB203580 pretreatment partly prevented these changes (Fig. 4B, C and D).

\section{Impact of p38 MAPK inhibition on the expression of ZO-1 and filtration barrier function of mouse podocytes incubated with CagA}

Selective p38 MAPK inhibition via SB203580 pretreatment significantly increased the mRNA level of ZO-1 which was suppressed by CagA treatment, although the level was still statistically different from the control group (Fig. 5A). Similar results were observed for the ZO-1 protein expression (Fig. 5B and C). Moreover, albumin influx assay showed that SB203580 pretreatment notably attenuated albumin influx across the podocyte
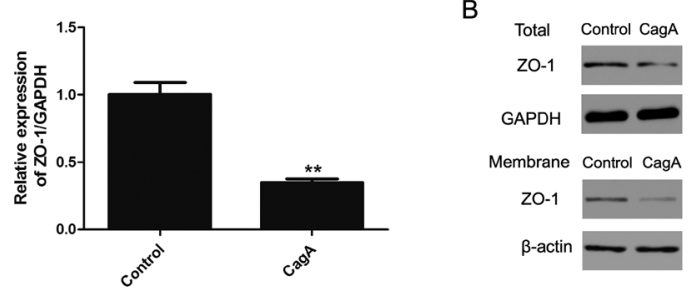

C
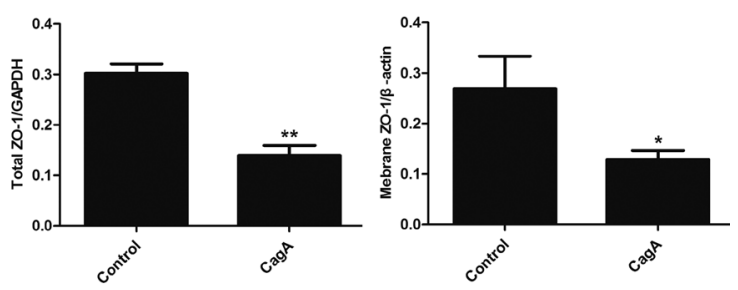

D

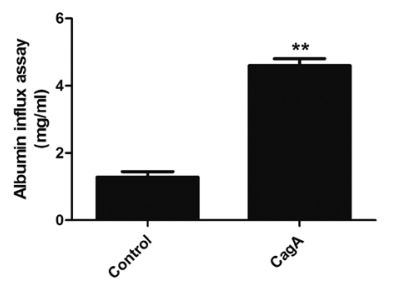

Figure 3. CagA decreased the expression and membrane distribution of ZO-1, impaired filtration barrier function of mouse podocytes at $72 \mathrm{~h}$ of incubation.

(A) Real time RT-PCR showed that when compared to control group, CagA significantly downregulated the mRNA expression of ZO-1. (B and C) Western blotting (B) and quantitative analysis (C) indicated that both the total and membrane ZO-1 protein expression was notably decreased by CagA treatment. (D) Albumin influx assay showed that CagA markedly increased albumin influx across the podocyte monolayer, which suggested podocyte filtration barrier injury. ${ }^{*} p<0.05$, compared to control; ${ }^{* *} p<0.01$, compared to control.

monolayer which was induced by CagA treatment, although it was still statistically different from the control group (Fig. 5D).

\section{DISCUSSION}

Among the virulence factors of $\mathrm{Hp}$, CagA remains the only one which translocates into cells via type IV secretion system (Jimenez-Soto et al, 2009; Odenbreit et al, 2000; Song et al., 2013). For the mechanisms of CagA delivery, recent study revealed that after being exposed on the bacterial surface via type IV secretion, CagA interacts with phosphatidylserine of host plasma membrane and then enters the host cells via an endocytic process distinct from known endocytic pathways (Murata-Kamiya et al., 2010). CagA was also found in serum-derived exosomes in patients with $\mathrm{Hp}$ infection, and CagA-containing exosomes can be internalized into cells via endocytosis or membrane fusion, independently of type IV secretion (Shimoda et al., 2016). Therefore, it is acceptable that, by directly interacting with phosphatidylserine of host plasma membrane, exogenous CagA can enter the cells via endocytosis. Actually, similarly to the present study, previous in vitro studies showed that recombinant CagA protein may directly influence cell viability or function (Gajewski et al., 2015; Lin et al., 2015; Wang et al., 2016).

CagA can regulate the expression of target proteins in a dose- and/or time-dependent manner (Brandt et al., 2005; Kang et al., 2013; Lina et al., 2013). However, both 
A

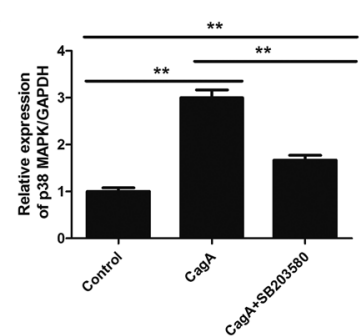

C

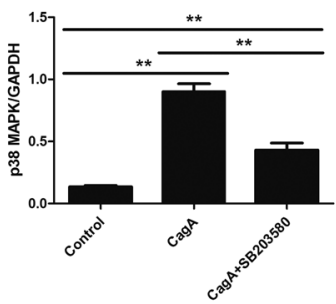

B

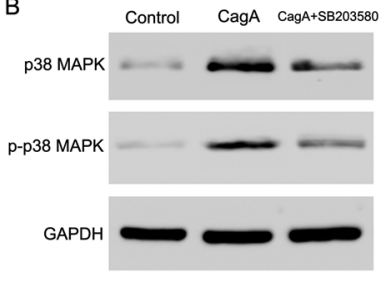

D

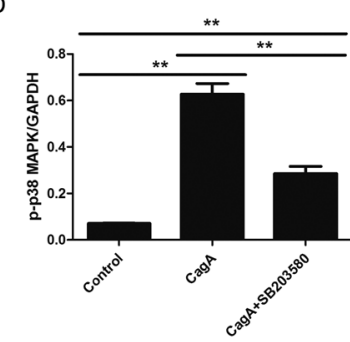

Figure 4. CagA promoted p38 MAPK expression and phosphorylation in mouse podocytes at $72 \mathrm{~h}$ of incubation.

(A) Real time RT-PCR showed that when compared to control, CagA significantly increased the p38 MAPK mRNA level, while pretreatment with SB203580 partly diminished this effect. (B) Western blotting analysis revealed that in comparison to control, CagA significantly upregulated p38 MAPK protein expression and phosphorylation (p-p38 MAPK), while pretreatment with SB203580 partly prevented these changes. (C and D) Quantitative analysis of relative protein level of p38 MAPK (C) and p-p38 MAPK (D) (normalized to GAPDH). ${ }^{*} p<0.05$, compared to control; ${ }^{* *} p<0.01$, compared to control.

unchanged and decreased ZO-1 expression were reported in gastric epithelial cells with CagA-positive Hp infection (Krueger et al., 2007; Zhang et al., 2014). While CagA-positive $\mathrm{Hp}$ infection activated $\beta$-catenin signaling in gastric epithelial cells, $\beta$-catenin inhibited the expression of ZO-1 in podocytes (Murata-Kamiya, 2011; Zhou et al., 2015). Therefore, it seems reasonable that CagA may have the potential to suppress ZO-1 expression via $\beta$-catenin. In the present study, we found that CagA could dose- and time-dependently decrease ZO-1 expression in mouse podocytes.

Tight junction protein ZO-1 located in the membrane surface of podocytes and playes an essential role in establishing podocyte filtration barrier ( $\mathrm{Ha}, 2013$; Itoh et al., 2014). Abnormal expression and/or distribution of ZO-1 may impair the filtration barrier function of podocytes and then induce proteinuria. In MWF rats, an animal model of spontaneous proteinuria, abnormal glomerular ZO-1 distribution alone may cause proteinuria (Ha, 2013). In animal model of diabetic kidney disease, high glucose decreases the expression and simultaneously alters the distribution of ZO-1 in glomerular epithelial cells, which is associated with proteinuria (Rincon-Choles et al., 2006). In the present study, we demonstrated that after CagA treatment, consistently with the reduced expression of total ZO-1, the distribution of membrane ZO-1 was also decreased, which consequently induced an increased albumin influx across the podocyte monolayer, resulting in an impaired filtration barrier function of mouse podocytes.

The p38 MAPK signaling pathway plays a crucial role in regulating cellular response (Cuadrado \& Nebreda, 2010). CagA-positive Hp infection or CagA transfection may induce p38 MAPK phosphorylation in gastric epithelial cells (Allison et al., 2009; Liu et al., 2012). In Hp-dependent gastric mucosa-associated lymphoid tissue (MALT) lymphoma, the expression level of CagA was closely associated with the activation of p38 MAPK

A

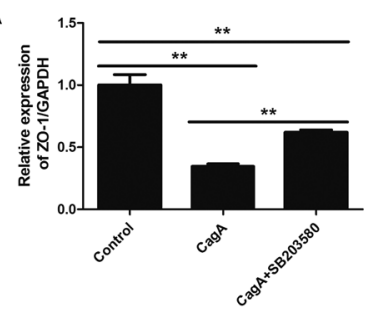

B

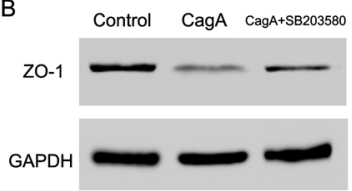

C
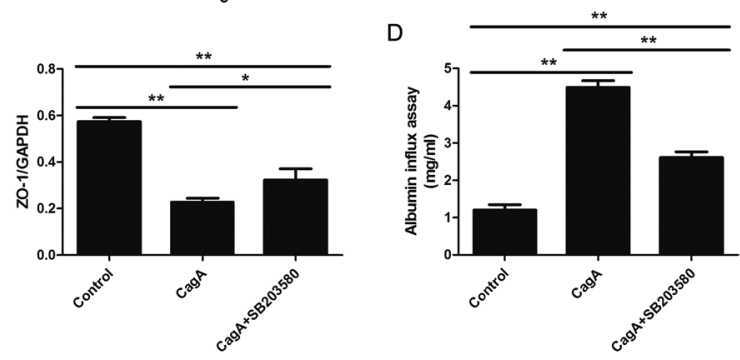

Figure 5. Selective p38 MAPK inhibition improved ZO-1 expression and filtration barrier function in mouse podocytes treated with CagA for $72 \mathrm{~h}$.

(A and B) Real time RT-PCR (A) and western blotting analysis (B) showed that pretreatment with SB203580 notably increased the mRNA level and protein expression of ZO-1 which was downregulated by CagA treatment. (C) Quantitative analysis of relative protein level of ZO-1 (normalized to GAPDH). (D) Albumin influx assay revealed that, when compared to CagA treatment, pretreatment with SB203580 significantly reduced albumin influx across the podocyte monolayer. ${ }^{*} p<0.05$, compared to control; ${ }^{* *} p<0.01$, compared to control.

(Kuo et al., 2015). In the present study, we also revealed that CagA promoted the expression and phosphorylation of p38 MAPK in mouse podocytes. On the other hand, consistently with the previous reports, SB203580 pretreatment significantly inhibited these changes (Hai-Yan et al., 2013; Ding et al., 2015).

P38 MAPK activation mediated calcium oxalate crystal-induced downregulation of ZO-1 in distal renal tubular epithelial cells and regulated the distribution of ZO-1 in cell-cell contacts of keratinocytes (Peerapen \& Thongboonkerd, 2013; Minakami et al., 2015). Both in human podocyte injury diseases and animal models of nephropathy, podocyte p38 MAPK signaling was activated and associated with proteinuria. P38 MAPK inhibition significantly ameliorated podocyte injury and suppressed proteinuria (Koshikawa et al., 2005). In the present study, we revealed that p38 MAPK inhibition by SB203580 pretreatment markedly improved CagA-induced downregulation of ZO-1 and consequently protected the filtration barrier function of mouse podocytes.

In summary, our results suggest that CagA, one of the key virulence factors of $\mathrm{Hp}$, decreases the expression and membrane distribution of tight junction protein ZO-1, impairs the filtration barrier function of podocytes, while activating p38 MAPK signaling pathway in these cells. Selective p38 MAPK inhibition partly prevents CagA-induced filtration barrier dysfunction of podocytes through ameliorating ZO-1 downregulation. Therefore, CagA, at least via p38 MAPK signaling pathway, may induce podocyte injury. Anti-Hp therapy may be beneficial for the treatment of kidney diseases related to Hp antigen deposition.

\section{Acknowledgements}

This study was supported by the Research Fund from Shanghai Municipal Commission of Health and Family Planning (Grant No. 20144Y0121). 


\section{REFERENCES}

Allison CC, Kufer TA, Kremmer E, Kaparakis M, Ferrero RL (2009) Helicobacter pylori induces MAPK phosphorylation and AP-1 activation via a NOD1-dependent mechanism. J Immunol 183: 8099-8109. http://doi: 10.4049/jimmunol.0900664

Brandt S, Kwok T, Hartig R, Konig W, Backert S (2005) NF-kappaB activation and potentiation of proinflammatory responses by the Helicobacter pylori CagA protein. Proc Natl Acad Sci US A 102: 93009305

Brinkkoetter PT, Ising C, Benzing T (2013) The role of the podocyte in albumin filtration. Nat Rev Nephrol 9: 328-336. http://doi: $10.1038 /$ nrneph.2013.78

Censini S, Lange C, Xiang Z, Crabtree JE, Ghiara P, Borodovsky M, Rappuoli R, Covacci A (1996) cag, a pathogenicity island of Helicobacter pylori, encodes type I-specific and disease-associated virulence factors. Proc Natl Acad Sci US A 93: 14648-14653

Covacci A, Censini S, Bugnoli M, Petracca R, Burroni D, Macchia G, Massone A, Papini E, Xiang Z, Figura N, et al. (1993) Molecular characterization of the $128-\mathrm{kDa}$ immunodominant antigen of Helicobacter pylori associated with cytotoxicity and duodenal ulcer. Proc Natl Acad Sci U S A 90: 5791-5795

Cuadrado A, Nebreda AR (2010) Mechanisms and functions of p38 MAPK signalling. Biochem J 429: 403-417. http://doi: 10.1042/ BJ20100323

Dede F, Ayli D, Gonul I, Yuksel O, Ozturk R, Yildiz A, Yenigun E, Piskinpasa S, Turgut D, Koc E, Odabas AR (2015) The effect of Helicobacter pylori eradication on proteinuria in patients with primary glomerulonephritis. Arch Med Sci 11: 764-769. http://doi: 10.5114/ aoms.2013.37013

Ding W, Jiang Y, Zhu T, Xu Y, Jiang W, Zhu W, Tang Z, Ge Z, Ma T, Tan Y (2015) Role of SB203580 in the regulation of human esophageal cancer cells under the effection of Diosgenin. Int J Clin Exp Med 8: 2476-2479

Eusebi LH, Zagari RM, Bazzoli F (2014) Epidemiology of Helicobacter pylori infection. Helicobacter 19 (Suppl 1): 1-5. http://doi: 10.1111/ hel.12165

Franceschi F, Tortora A, Gasbarrini G, Gasbarrini A (2014) Helicobacter pylori and extragastric diseases. Helicobacter 19 (Suppl 1): 52-58. http://doi: 10.1111/hel.12159

Gajewski A, Mnich E, Szymański K, Hinc K, Obuchowski M, Moran AP, Chmiela M (2016) Helicobacter pylori antigens, acetylsalicylic acid, LDL and 7-ketocholesterol - their potential role in destabilizing the gastric epithelial cell barrier. An in vitro model of Kato III cells. Acta Biochim Pol 63: 145-152. http://doi: 10.18388/abp.2015_1122

Ha TS (2013) Roles of adaptor proteins in podocyte biology. World J Nephrol 2: 1-10. http://doi: 10.5527/win.v2.i1.1

Hai-Yan Z, Yong-Hong G, Zhi-Yao W, Bing X, Ai-Ming W, Yan-Wei X, Bei L, Li-Xia L, Li-Xin C (2013) Astragalus polysaccharide suppresses the expression of adhesion molecules through the regulation of the p38 MAPK signaling pathway in human cardiac microvascular endothelial cells after ischemia-reperfusion injury. Evid Based Complement Alternat Med 2013: 280493. http://doi: 10.1155/2013/280493

Itoh M, Nakadate K, Horibata Y, Matsusaka T, Xu J, Hunziker W, Sugimoto $H$ (2014) The structural and functional organization of the podocyte filtration slits is regulated by Tjp1/ZO-1. PLoS One 9: e106621. http://doi: 10.1371/journal.pone.0106621

Jimenez-Soto LF, Kutter S, Sewald X, Ertl C, Weiss E, Kapp U, Rohde M, Pirch T, Jung K, Retta SF, Terradot L, Fischer W, Haas R (2009) Helicobacter pylori type IV secretion apparatus exploits beta1 integrin in a novel RGD-independent manner. PLoS Pathog 5: e1000684. http://doi: 10.1371/journal.ppat.1000684

Jing Z, Wei-jie Y, Yi-Feng ZG (2015) Down-regulation of Wt1 activates Wnt/beta-catenin signaling through modulating endocytic route of LRP6 in podocyte dysfunction in vitro. Cell Signal 27: 17721780. http://doi: 10.1016/j.cellsig.2015.05.018

Kang DW, Hwang WC, Park MH, Ko GH, Ha WS, Kim KS, Lee YC, Choi KY, Min DS (2013) Rebamipide abolishes Helicobacter pylori CagA-induced phospholipase D1 expression via inhibition of NFkappaB and suppresses invasion of gastric cancer cells. Oncogene 32: 3531-3542. http://doi: 10.1038/onc.2012.358

Koshikawa M, Mukoyama M, Mori K, Suganami T, Sawai K, Yoshioka T, Nagae T, Yokoi H, Kawachi H, Shimizu F, Sugawara A, Nakao $\mathrm{K}$ (2005) Role of p38 mitogen-activated protein kinase activation in podocyte injury and proteinuria in experimental nephrotic syndrome. J Am Soc Nephrol 16: 2690-2701

Krueger S, Hundertmark T, Kuester D, Kalinski T, Peitz U, Roessner A (2007) Helicobacter pylori alters the distribution of ZO-1 and p120ctn in primary human gastric epithelial cells. Pathol Res Pract 203: $433-444$
Kumar PA, Kotlyarevska K, Dejkhmaron P, Reddy GR, Lu C, Bhojani MS, Menon RK (2010) Growth hormone (GH)-dependent expression of a natural antisense transcript induces zinc finger E-boxbinding homeobox 2 (ZEB2) in the glomerular podocyte: a novel action of gh with implications for the pathogenesis of diabetic nephropathy. J Biol Chem 285: 31148-31156. http://doi: 10.1074/jbc. M110.132332

Kuo SH, Yeh KH, Chen LT, Lin CW, Hsu PN, Wu MS, Liou JM, Tsai HJ, Tzeng YS, Cheng AL (2015) Helicobacter pylori CagA translocation is closely associated with the expression of CagA-signaling molecules in low-grade gastric mucosa-associated lymphoid tissue lymphoma. Am I Surg Pathol 39: 761-766. http://doi: 10.1097/ PAS.0000000000000437

Li Q, Lin X, Wu Z, He L, Wang W, Cao Q, Zhang J (2013) Immunohistochemistry analysis of Helicobacter pylori antigen in renal biopsy specimens from patients with glomerulonephritis. Saudi J Kidney Dis Transpl 24: 751-758

Lina TT, Pinchuk IV, House J, Yamaoka Y, Graham DY, Beswick EJ, Reyes VE (2013) CagA-dependent downregulation of B7-H2 expression on gastric mucosa and inhibition of Th17 responses during Helicobacter pylori infection. I Immunol 191: 3838-3846. http://doi: 10.4049 /jimmunol.1300524

Liu Z, Xu X, Chen L, Li W, Sun Y, Zeng J, Yu H, Chen C, Jia J (2012) Helicobacter pylori CagA inhibits the expression of Runx3 via Src/MEK/ERK and p38 MAPK pathways in gastric epithelial cell. I Cell Biochem 113: 1080-1086. http://doi: 10.1002/jcb.23440

Minakami M, Kitagawa N, Iida H, Anan H, Inai T (2015) p38 Mitogen-activated protein kinase and c-Jun NH2-terminal protein kinase regulate the accumulation of a tight junction protein, ZO-1, in cell-cell contacts in HaCaT cells. Tissue Cell 47: 1-9. http://doi: 10.1016/j.tice.2014.10.001

Murata-Kamiva N (2011) Pathophysiological functions of the CagA oncoprotein during infection by Helicobacter pylori. Microbes Infect 13: 799-807. http://doi: 10.1016/j.micinf.2011.03.011

Murata-Kamiya N, Kikuchi K, Hayashi T, Higashi H, Hatakeyama M (2010) Helicobacter pylori exploits host membrane phosphatidylserine for delivery, localization, and pathophysiological action of the CagA oncoprotein. Cell Host Microbe 7: 399-411. http://doi: 10.1016/j. chom.2010.04.005

Nagashima R, Maeda K, Yuda F, Kudo K, Saitoh M, Takahashi T (1997) Helicobacter pylori antigen in the glomeruli of patients with membranous nephropathy. Virchows Arch 431: 235-239

Odenbreit S, Puls J, Sedlmaier B, Gerland E, Fischer W, Haas R (2000) Translocation of Helicobacter pylori CagA into gastric epithelial cells by type IV secretion. Science 287: 1497-500

Peerapen P, Thongboonkerd V (2013) p38 MAPK mediates calcium oxalate crystal-induced tight junction disruption in distal renal tubular epithelial cells. Sci Rep 3: 1041. http://doi: 10.1038/srep01041

Rincon-Choles H, Vasylyeva TL, Pergola PE, Bhandari B, Bhandari K, Zhang JH, Wang W, Gorin Y, Barnes JL, Abboud HE (2006) ZO-1 expression and phosphorylation in diabetic nephropathy. Diabetes 55: $894-900$

Song X, Chen HX, Wang XY, Deng XY, Xi YX, He Q, Peng TL, Chen J, Chen W, Wong BC, Chen MH (2013) pylori-encoded CagA disrupts tight junctions and induces invasiveness of AGS gastric carcinoma cells via $\mathrm{Cdx} 2$-dependent targeting of Claudin-2. Cell Immunol 286: 22-30. http://doi: 10.1016/j.cellimm.2013.10.008

Testerman TL, Morris J (2014) Beyond the stomach: an updated view of Helicobacter pylori pathogenesis, diagnosis, and treatment. World J Gastroenterol 20: 12781-12808. http://doi: 10.3748/wig.v20.i36.12781

Wang L, Tan RZ, Chen Y, Wang HL, Liu YH, Wen D, Fan JM (2016) CagA promotes proliferation and secretion of extracellular matrix by inhibiting signaling pathway of apoptosis in rat glomerular mesangial cells. Ren Fail 38: 458-464. http://doi: 10.3109/0886022X.2016.1138831

Yang AH, Lin BS, Kuo KL, Chang CC, Ng YY, Yang WC (2009) The clinicopathological implications of endothelial tubuloreticular inclusions found in glomeruli having histopathology of idiopathic membranous nephropathy. Nephrol Dial Transplant 24: 3419-3425. http:// doi: $10.1093 / \mathrm{ndt} / \mathrm{gfp} 288$

Zhang C, Zhang H, Yu L, Cao Y (2014) Helicobacter pylori dwelling on the apical surface of gastrointestinal epithelium damages the mucosal barrier through direct contact. Helicobacter 19: 330-342. http:// doi: 10.1111/hel.12138

Zhou L, Li Y, He W, Zhou D, Tan RJ, Nie J, Hou FF, Liu Y (2015) Mutual antagonism of Wilms' tumor 1 and beta-catenin dictates podocyte health and disease. J Am Soc Nephrol 26: 677-691. http://doi: 10.1681/ASN.2013101067 